\title{
A Scripting Language for Multimodal Presentation on Mobile Phones
}

\author{
Santi Saeyor $^{1}$, Suman Mukherjee ${ }^{2}$, Koki Uchiyama ${ }^{2}$, Ishizuka Mitsuru ${ }^{1}$ \\ ${ }^{1}$ Dept. of Information and Communication Engineering, University of Tokyo, \\ Japan \{santi,ishizuka\}@miv.t.u-tokyo.ac.jp \\ 2 Hottolink Inc., Japan \{uchi,suman\}@hottolink.co.jp
}

\begin{abstract}
Mobile phones have become indispensible devices. At the same time, their computing capabilities and graphical resources keep getting higher until 3D embodied character agent with attractive movements can be realized. In order to get this rich data presentation to target users, however, the author needs to deal with various programming works. In this paper, we propose a simple scripting language that keeps difficult works from ordinary user but allows sufficient attractive character controls.
\end{abstract}

\section{Introduction}

Mobile phones have become indispensable device in daily life. At the same time, their functions are increasing both in voice and non-voice services. Thanks to the increasing capabilities, embodied character agent is now available on mobile phones via dedicated 3D graphical engine in Japanese mobile phones.

We have studied the possibility of bringing multimodal presentation to this platform as a successive work to MPML (Multimodal Presentation Markup Language) introduced by Ishizuka et al. MPML has been developed and released on PC based computing environment. Some breeds of MPML are implemented using Microsoft Agents with emotional computing in the backend [3]. There was a version of MPML that uses embodied character agents in VRML (Virtual Reality Modeling Language) world. [1] Moreover, MPML has been used for scripting presentation that made by life-like agents on the web. [2][4] 
However, we have found that the original specifications are impossible to be implemented on mobile phones due to its limited computational and graphical resources. This paper describes the issues as well as their solutions when dealing with mobile phones. It also demonstrates how to implement a lighter specification of MPML in order to make the most out of using MPML on mobile phones.

\section{Multimodal Presentation Markup Language: MPML}

MPML is a markup language, which is designed and developed to facilitate multimodal presentation by character agents. It has the following features:

- Platform Independent: it is designed so that the contents written in MPML can be played on wide variety of tools and players.

- Simplicity: MPML conforms to XML (Extensible Markup Language) specification. MPML version 1.0 has 19 tags. For those who can write HTML scripts to build web pages, they will find that writing multimodal presentation by character agents in MPML is quite simple.

- Media Synchronization: Synchronization of media such as voices, images and gestures is necessary to create an attractive presentation. On this purpose, W3C announced SMIL (Synchronized Multimedia Integration Language), which is a language for controlling complex media data on WWW in 1998. MPML implements media synchronization in the same way.

- Controls of Character Agents : MPML supports action controls of character agents such as greeting, pointing and explaining.

- Controls of Interactive Presentation: Simple interactions are supported.

\section{Comparison with Other Markup Languages}

The comparison of MPML with other markup languages (SMIL and HTML) is shown in (Table 2).

Even all these markup languages are designed for Web publication, there are some differences. For example, since SMIL is designed mainly for media synchronization, the description of layout and timing for playing the media are strengthened in its specification. On the other side, since MPML is designed mainly for simplicity in character agent based multimodal presentation content composing, it incorporates only minimum media synchronization and layout features sufficient to perform presentation. Furthermore, due to the need of speech dialogue features, it has to incorporate voice commands and TTS (Text-ToSpeech) capability. 


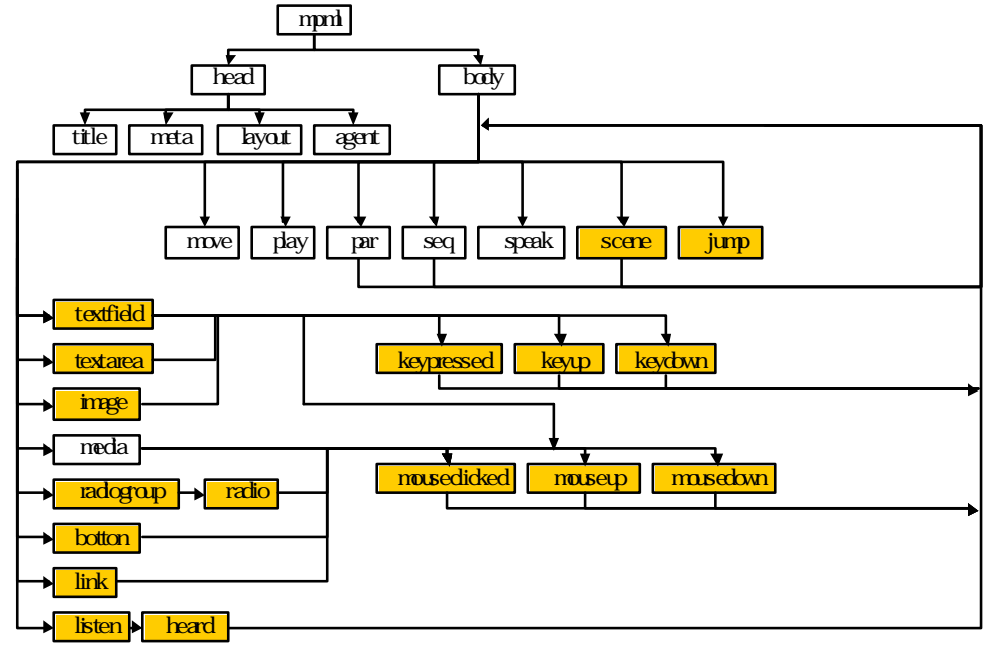

Added inMPM 20

Figure 1: MPML structure tree

Table 2: Comparison of MPML with other WWW markup languages.

\begin{tabular}{|llll|}
\hline Scripting Function & MPML & SMIL & HTML \\
Web publication & Possible & Possible & Possible \\
Link to other URLs & Possible & Possible & Possible \\
Media Synchronization & Minimum features & Full features & Impossible \\
Agent's action description & Possible & Impossible & Impossible \\
Mouse Control & Possible & Possible & Possible \\
Voice Control & Possible & Impossible & Impossible \\
Text to speech & Possible & Impossible & Impossible \\
Current users & Very little & Few & Remarkably large \\
Tools & Few & A great number & A great number \\
Number of tags & About 30 & About 20 & About 80 \\
\hline
\end{tabular}

\section{Original MPML is not ready for mobile phones}

MPML doesn't define how to control character agent behavior from the user's actions on various objects/components inside a web page. For example, if we require the action of a character agent to change when a user clicks on a link / button, we cannot achieve that using just MPML. It doesn't define how to "get", "set" or "unset" the values of these variables from inside the web pages. 


\section{Making MPML mobile edition out of MPML}

There are two aspects of MPML that MPML mobile edition inherits, albeit by a different design:

\section{Sequential Flow of scene: Natural and Dynamic}

By invoking $<$ goto $>$ tags inside the web page, we can refer to different MPML scene sequentially. By default the scenes are executed in the order in which they appear inside the web page markup. This is the Natural or default order of scene execution. If the agent has to interact with the user inside a scene to select the next scene, a Dynamic flow happens. $<$ ask $>$ tags with any number of $<$ choice $>$ child tags can be used to achieve that. By putting appropriate values for the attributes 'url', 'scene' and 'how', it is possible to move to:

a) A new web page at the background with the agent not executing the remaining scenes of the old page and starting off with the first scene of the new page.

b) A new web page at the background with the agent continuing with its remaining scenes in the old page, discarding the scenes of the new page.

c) Staying at the same web page with the agent changing to a new scene in the current page.

d) Staying at the same web page with the agent changing to a subscene of the current scene.

\section{Variables}

User can be asked a question and the answer typed by the user can be stored for subsequent usage. Three levels of storage can be defined:

- Global

- Session

- Local

The "Global" scope variables are stored persistently and, once defined, all the scenes in all the pages across all future sessions of this application can access them. The "Session" scope variables are stored persistently across the current session. This means, the "Session" scope variables, once defined, can be used by all the subsequent pages in the current session. The "Local" scope variables can be used only in the scenes of the current page. Redefined "Local" and "Session" variables are over-written. "Global" variables, once defined, cannot be redefined. This is due to a security threat for global variables. For example, if the global variable storing the user name could be re-written, it may be easy to track its name and change its value to something different, like abusive phrases, in another MPML page. 


\section{Controlling character-agent based multimodal presentation}

A set of action tags, defined earlier enables control of agent behavior. The execution of the actions is restricted to sequential, due to limitations in CLDC (Connected Limited Device Configuration) devices. The 'act'-s relevant to a particular agent is entirely dependent on the browser implementation. The speed of character agent's movement or change of posture is dependent on the processing power of the respective machines and the browser implementation.

\section{Controlling character's behavior from user's action on form components /} links

In addition, 'MPML mobile edition' has features that deal with 'change of character behavior with user's action on web page components or objects.' An approach similar to the JavaScript approach has been taken for this purpose. 'Extended' attributes for HTML Form components and 'Link' tags have been defined for user events like OnClick, onSelect, onDeSelect and onChange on the web page. The extended attributes are as follows:

- onClickGoTo

- onSelectGoTo

- onDeSelectGoTo

- onChangeGoTo

The value of each of these attributes is a valid scene ID or a valid subscene ID in the same page. They make the character jump to the given scene in case of the following respective events: OnClick, onSelect, onDeSelect, onChange.

\section{MPML mobile edition's Tag Specification}

MPML mobile edition also conforms to XML but was rearranged to suit mobile phones' capabilities. The tag specifications are shown in the Table 1.

Table 1. MPML mobile edition's tag specification

\begin{tabular}{|l|l|c|l|}
\hline Tag & Attribute & Value & \multicolumn{1}{|c|}{ Description } \\
\hline Mpml & None & NA & Top element. \\
\hline Scene & id & Any string. & Unique Identifier. \\
\hline Show & chara & Character id. & Act on this character. \\
\hline Hide & chara & Character $i d$. & Act on this character. \\
\hline & chara & Character $i d$. & Act on this character. \\
\hline
\end{tabular}


Santi Saeyor1, Suman Mukherjee2, Koki Uchiyama2, Ishizuka Mitsuru1

\begin{tabular}{|c|c|c|c|}
\hline Play & chara & Character id. & Act on this character. \\
\hline \multirow{3}{*}{$\begin{array}{l}\text { Mayce_1 } \\
\text { (Walk) }\end{array}$} & abara & Qhomas tef aidl. & Ehonat this abaracter. \\
\hline & $\mathrm{x}$ & supported stakes & Move to this $\mathrm{x}$-coordinate. \\
\hline & $\mathrm{y}$ & Positive Integer. & Move to this $y$-coordinate. \\
\hline \multirow{3}{*}{$\begin{array}{l}\text { Move_2 } \\
\text { (Fly) }\end{array}$} & chara & Character id. & Move this character. \\
\hline & $\mathrm{x}$ & Positive integer. & Move to this $\mathrm{x}$-coordinate. \\
\hline & $\mathrm{y}$ & Positive integer. & Move to this y-coordinate. \\
\hline \multirow{2}{*}{ Speak } & chara & Character id. & Make this character speak. \\
\hline & [Node value] & Any string. & Speak these words. \\
\hline \multirow{2}{*}{ Wait } & chara & Character id. & Make this character wait. \\
\hline & time & Time in seconds. & Wait for this time. \\
\hline \multirow[t]{4}{*}{ Ask } & chara & Character id. & Make this character ask. \\
\hline & scene & Scene Id. & $\begin{array}{l}\text { Pass on to this scene in this page } \\
\text { after user inputs answer or select } \\
\text { choice. }\end{array}$ \\
\hline & var & Any String. & $\begin{array}{l}\text { Name of the variable, if the } \\
\text { answer is to be stored. }\end{array}$ \\
\hline & scope & $\begin{array}{c}\text { "global" | } \\
\text { "session"| "local" }\end{array}$ & $\begin{array}{l}\text { The scope of the variable. "local" } \\
\text { by default. }\end{array}$ \\
\hline \multirow[t]{3}{*}{ GoTo } & url & URL path. & Go to this page. \\
\hline & Scene & Scene Id. & $\begin{array}{l}\text { Go to this scene of the same page } \\
\text { if no url is provided, else go to } \\
\text { this scene of new page. }\end{array}$ \\
\hline & how & $\begin{array}{r}\text { 'jump’|'html’ } \\
\text { (default is'jump') }\end{array}$ & $\begin{array}{l}\text { If 'jump', do not execute the } \\
\text { remaining actions in the current } \\
\text { scene and jump onto the first } \\
\text { scene of the next page. Else, only } \\
\text { change the background HTML } \\
\text { display and continue with current } \\
\text { list of character behavior. }\end{array}$ \\
\hline \multirow[t]{5}{*}{ SubScene } & id & Subscene id. & Scene within a scene. \\
\hline & url & URL path. & $\begin{array}{l}\text { Go to this URL if this choice is } \\
\text { selected. }\end{array}$ \\
\hline & scene & Scene Id. & $\begin{array}{l}\text { If this choice is selected, go to } \\
\text { this scene of the same page if no } \\
\text { url is provided, or go to this scene } \\
\text { of the new page if url is provided. }\end{array}$ \\
\hline & how & $\begin{array}{r}\text { 'jump’|' 'html' } \\
\text { (default is' jump') }\end{array}$ & $\begin{array}{l}\text { If 'jump', do not execute the } \\
\text { remaining actions in the current } \\
\text { scene and jump onto the first } \\
\text { scene of the next page. Else, only } \\
\text { change the background HTML } \\
\text { display and continue with current } \\
\text { list of character behavior. }\end{array}$ \\
\hline & [Node value] & Any String. & The choice. \\
\hline
\end{tabular}




\section{Implementation}

The prototype of MPML player on mobile phones has been created using Java application for Japanese mobile handsets based on J2ME (Java 2 Mobile Edition). The application consists of MPML parser, 3D character agent controller, and dialog controller. The handset fetches the MPML data from a content server that keeps refreshing news every 30 minutes. The news headlines are reformatted into MPML mobile edition document that contains both content and character's controls. Figure 2 shows the playback of the MPML mobile document on a mobile phone in NTT Docomo's 504i series. [5]

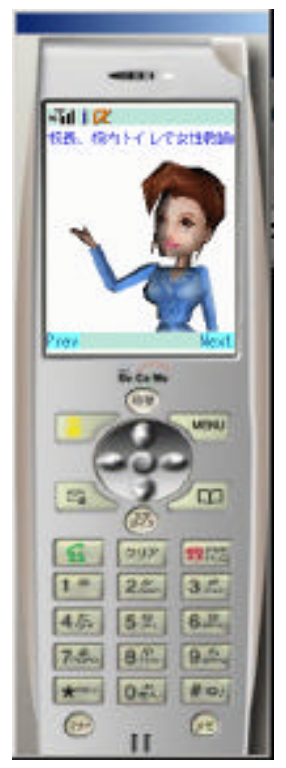

Figure 2: Implementation on mobile phone

The MPML parser is an XML parser with a very small footprint of $3 \mathrm{~KB}$ in size. We achieve this small profile because we assume that the MPML fed to the parser must be well formed XML so we can neglect a lot of code for error handling in the parsing process.

The character agent controller is a module that manipulates $3 \mathrm{D}$ engine inside the mobile phone. At the moment, the character agent and its animations must be created before hand. Its animation must be pre-defined and packaged with the model data. We have about 15 animations for the character in this package. This has to be limited to a small number of actions because the animation data will occupy a lot of space in Java package (JAR file) and reaches the limit of handset specification (about $30 \mathrm{~KB}$ ). However, the available actions are adequate for showing affective behaviors and movements in presenting the news headlines to the users. 
The dialog controller controls the display of message spoken by the character agent. We use the sliding text, which occupies only one line on the small display of mobile phones, in order to make spaces for character agent and background images.

\section{Conclusion}

This paper shows the approaches we used to bring multimodal presentation to mobile phones based on MPML mobile edition.

The MPML mobile edition also conforms to XML specification but was arranged specially for limited device like mobile phones. Player software that interprets MPML document can be created with small footprint because of simple specification of MPML mobile edition. When combined with 3D character engine in mbile handsets, we successfully realized multimodal presentation system on mobile phone platforms.

The use of MPML mobile edition is not limited to the prototype shown in this paper. It can also be used in mobile educational system. The affective behaviors and attractive movements could be of any help to attract people to view the content that we send in MPML document. However, there are still some issues such as speed, data packet's cost, authoring tools, etc. that are left to be solved. These will be tackled in our successive work.

\section{Reference}

[1] Okazaki, N., S.Aya, S. Saeyor, and M. Ishizuka. 2002. A Multi-modal Markup Language MPML-VR for 3D virtual space. In: Proceedings (CD-ROM) of Workshop on Virtual Conversational Characters: Applications, Methods, and Research Challenges (in conj. With HF2002 and OZCHI2002)

[2] Prendiger, H., S. Descamps, and M. Ishizuka. 2002. Scripting affective communication with life-like characters in web-based interaction systems. Applied Artificial Intelligence, 16:519-553.

[3] Prendinger, H. and M. Ishizuka. 2001 Social role awareness in animated agents. In: Proceeds $5^{\text {th }}$ International Conference on Autonomous Agents (Agents-01), 270-277. ACM Press.

[4] Saeyor. S. 2002. Multi-modal Presentation Markup Language Ver. 2.2a (MPML2.2a) URL: http://www.miv.t.u-tokyo.ac.jp/ santi/research/mpml2a

[5] Saeyor. S. 2002. 3D News Caster for iAppli on NTT Docomo's 504i series and JPhone's Java Appli http://www.miv.t.u-tokyo.ac.jp/i/3Dcaster 